\title{
A Method for Calculating Inverse Trigonometric Functions
}

Introduction. The problem of calculating an angle from a known trigonometric function of that angle occurs frequently in the numerical analysis of physical systems. The purpose of this paper is to introduce a new method which is particularly suitable for the I. B. M. Card Programmed Calculator.

Methods. There are several methods of determining an angle from its trigonometric functions; the more commonly employed ones are:

1. Table look up.

2. Infinite processes

(a) Power series

(b) Continued fractions. ${ }^{1}$

3. Approximations. ${ }^{2}$

Which of these methods is the best to use depends primarily on the computing equipment that is to be used. For desk calculating, tables are usually best; for computers with limited storage capacity, the infinite processes are usually used; on large scale computers, the approximations are most efficient. Since the IBM-CPC has a very limited amount of high speed storage, the common practice has been to use a Maclaurin's series with a variable number of terms. ${ }^{3}$ Other methods have been devised ${ }^{4}$ that speed up the process by first transforming the argument into a smaller number so that the series will converge more rapidly.

The New Method. The above discussion helps to illustrate the problem facing the programmer when programming a method for determining an angle from its trigonometric function. An entirely different method for finding inverse functions will now be described. This method is more of a logical process than a mathematical one. The following notation will be used :

$$
y=\operatorname{trig}^{-1}(A, B)
$$

which reads, " $y$ is the inverse trigonometric function of $A$ and $B$. ." Furthermore,

$$
\begin{aligned}
& A=\sin y, \\
& B=\cos y .
\end{aligned}
$$

In the case both the sine and cosine of $y$ may be found independently, this method will determine the angle in its proper quadrant. If the cosine must be found by an identity from the sine, then only the principal value will be computed.

Let us assume that the sine and cosine of some angle, $y$, is known and it is subject to the following restrictions:

$$
\begin{gathered}
0<y<\pi \\
y \neq \frac{\pi}{2^{m}} \quad(m=0,1,2, \cdots, 25) .
\end{gathered}
$$

The excluded values are special cases and will be discussed later. We first calculate $\sin 2 y$ and $\cos 2 y$ by suitable trigonometric formulae. From the 
first restriction, $0<y<\pi$, we have $\sin y>0$; if

$$
\sin 2 y>0 \text {, then } 0<2 y<\pi \text { and } 0<y<\frac{\pi}{2} \text {, }
$$

but if

$$
\sin 2 y<0, \text { then } \pi<2 y<2 \pi \text { and } \frac{\pi}{2}<y<\pi .
$$

It is obvious then that a balance test of $\sin 2 y$ will determine the quadrant of $y$; this information may be retained by storing the initial value of the quadrant. Calling the stored quantity $\Sigma_{1}$, it is seen that if

but if

$$
0<y<\frac{\pi}{2} \text {, then } \Sigma_{1}=0,
$$

$$
\frac{\pi}{2}<y<\pi \text {, then } \Sigma_{1}=\frac{\pi}{2} .
$$

From $\sin 2 y$ and $\cos 2 y$ we compute $\sin 4 y$ and $\cos 4 y$ and analyze in the same manner.

If $\sin 2 y>0$ and $\sin 4 y>0$, then $0<y<\frac{\pi}{4}$ and $\Sigma_{2}=0$,

if $\sin 2 y>0$ and $\sin 4 y<0$, then $\frac{\pi}{4}<y<\frac{\pi}{2} \quad$ and $\quad \Sigma_{2}=\frac{\pi}{4}$,

if $\sin 2 y<0$ and $\sin 4 y>0$, then $\frac{\pi}{2}<y<\frac{3 \pi}{4}$ and $\Sigma_{2}=\frac{\pi}{2}$,

if $\sin 2 y<0$ and $\sin 4 y<0$, then $\frac{3 \pi}{4}<y<\pi$ and $\Sigma_{2}=\frac{3 \pi}{4}$,

where $\Sigma_{2}$ is the initial value of the octant in which $y$ lies. A comparison of the $\Sigma_{2}$ 's and $\Sigma_{1}$ 's will show that when

and when

$$
\sin 4 y>0, \text { then } \Sigma_{2}=\Sigma_{1}
$$

$$
\sin 4 y<0, \text { then } \Sigma_{2}=\Sigma_{1}+\frac{\pi}{4} .
$$

In fact, if the same analysis is extended the following generalization will be apparent:

When

and when

$$
\sin 2^{n} y>0, \text { then } \Sigma_{n}=\Sigma_{n-1},
$$

$$
\sin 2^{n} y<0, \text { then } \Sigma_{n}=\Sigma_{n-1}+\frac{\pi}{2^{n}} .
$$

The $\Sigma_{n}$ will be the initial value of the interval, of width $\pi / 2^{n}$, in which $y$ lies. If this process is continued until $\pi / 2^{n}$ is less than the maximum allowable error, then $\Sigma_{n}$ will be the value of $y$. If the error is to be less than $5 \times 10^{-8}$ then $n$ must take on all values up to 26 . 
The cases where $y=\pi / 2^{m}$ cause difficulty because

$$
\sin 2^{n} y=0 \quad \text { if } n \geqq m .
$$

Actually, the number $\Sigma_{n}$ is the answer, $y$, when $n=m$ since $\Sigma_{n}=\Sigma_{m}=\frac{\pi}{2^{m}}$. Therefore, the first zero should be treated as a "minus" and all subsequent zeroes as "plus".

If

then

$$
y=0 \text { or } y=\pi
$$

and

$$
\sin y=0
$$

$$
\cos y=1 \text { or } \cos y=-1 \text {; }
$$

therefore, if $\sin y$ is zero, then a separate test must be made on the cosine to determine if $y=0$ or $y=\pi$.

The discussion thus far has assumed that $y$ is in the first two quadrants; the method can be extended to third and fourth quadrant angles by three methods:

1. The absolute value of $\sin y$ may be used and the final answer multiplied by minus one if $\sin y$ was originally negative. This gives answers in the range $-\pi<y \leqq \pi$.

2. If $\sin y$ is negative, set $\Sigma_{0}=\pi$; in the previous discussion it was implied that $\Sigma_{0}=0$. This will give answers in the range $0 \leqq y<2 \pi$.

3. If $\sin y$ is negative, set $\Sigma_{0}=-\pi$. This puts the same limits on $y$ as method 1 .

In most cases, either method 1 or method 3 is preferable to method 2.

Method 1 is illustrated in the flow diagram, Figure 1 . In practice it is more convenient to store $\sigma_{n}=\Sigma_{n} / \pi$ and $p_{n}=1 / 2^{n}$ instead of $\Sigma_{n}$ and $\pi / 2^{n}$, respectively. Since $p_{n}=\frac{1}{2} p_{n-1}$, any rounding error will be positive and the sum $\sigma_{n}$ will include the errors of all $p_{k}$ 's where $k \leq n$. This error may be as large as six in the last digit. This error may be reduced by always rounding to an even number or by carrying an extra digit in $p_{n}$ and $\sigma_{n}$; obviously both methods have disadvantages. The following is still another way in which the rounding error may be reduced and also save storage space. Let $a_{n}=0$ or $a_{n}=1$, depending on whether the term is to be omitted or added; also let $n=0,1,2, \cdots, N$; then

and

$$
y=\Sigma_{N}=\pi \sigma_{N}
$$

$$
\sigma_{N}=\frac{a_{1}}{2}+\frac{a_{2}}{4}+\frac{a_{3}}{8}+\cdots+\frac{a_{N}}{2^{N}}
$$

factoring out $\frac{1}{2^{N}}$, then

$$
\sigma_{N}=\frac{1}{2^{N}}\left(a_{1} 2^{N-1}+a_{2} 2^{N-2}+a_{3} 2^{N-3}+\cdots+a_{N}\right)
$$

or

$$
2^{N} \sigma_{N}=\left\{\left\{\left[\left(2 a_{1}+a_{2}\right) 2+a_{3}\right] 2+a_{4}\right\} 2+\cdots+a_{N}\right\} .
$$


A METHOD FOR CALCULATING INVERSE TRIGONOMETRIC FUNCTIONS

135

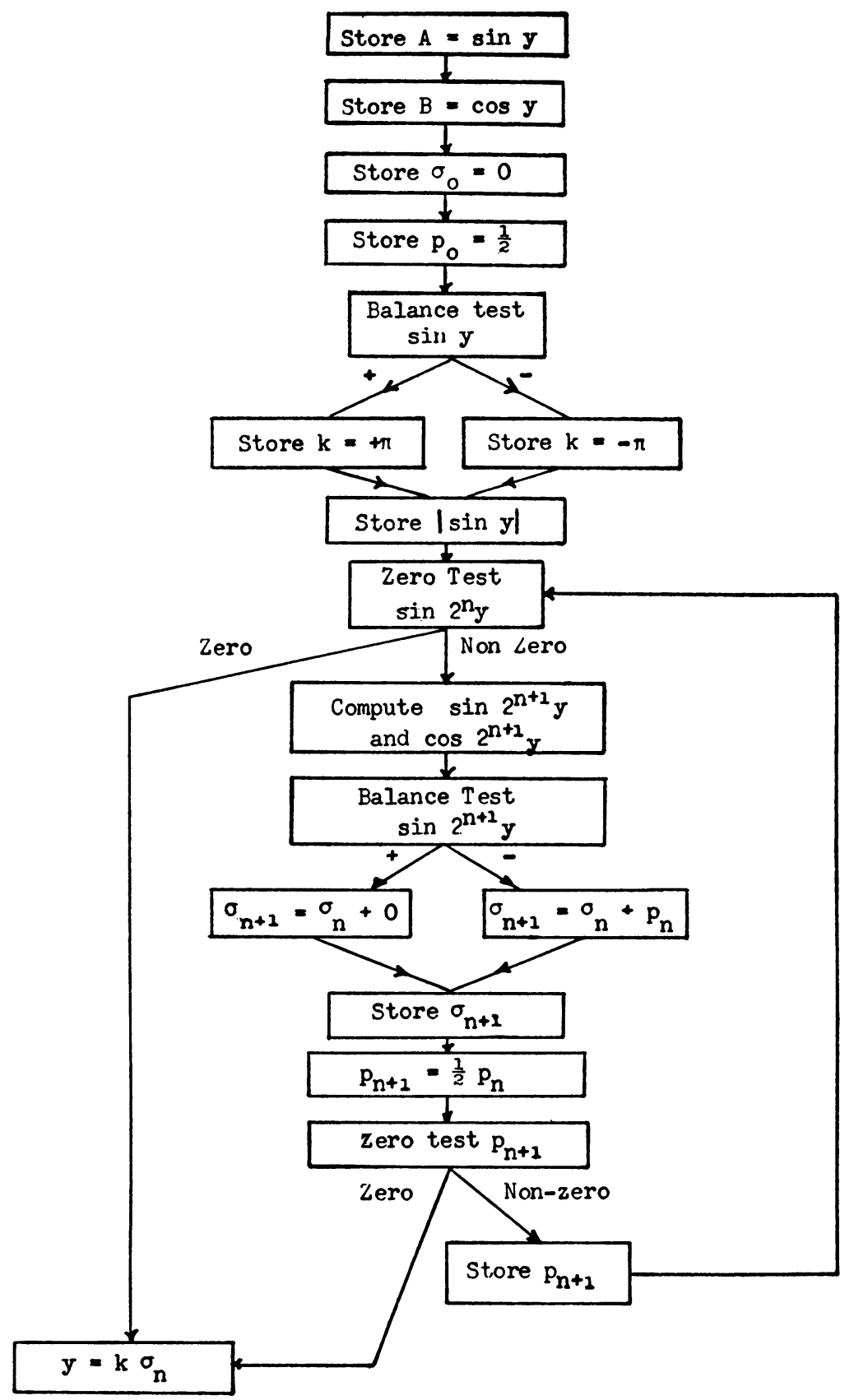

FIG. 1. 
The quantity $Z_{N}=2^{N} \sigma_{N}$ may be computed by the following formula:

$$
Z_{n+1}=2 Z_{n}+a_{n+1}
$$

where $Z_{0}=0$ and $n$ has the same meaning as before. The quantity thus computed is a whole number and is therefore exact, the only rounding error will be in the final multiplication by $\pi / 2^{N}$. The number of times $Z$ has been computed is subtracted from $N$ so that the process may be stopped when this quantity, $b_{n}$, is equal to zero. This also saves storage space since only two digits of $N$ need to be stored rather than seven or more of $p_{n}$. The flow diagram illustrating this method is shown in Figure 2.

In computing $\sin 2^{n+1} y$ and $\cos 2^{n+1} y$ both $\sin 2^{n} y$ and $\cos 2^{n} y$ must be used in each; otherwise the condition

$$
\left(\sin 2^{n+1} y\right)^{2}+\left(\cos 2^{n+1} y\right)^{2}=1
$$

will not always be satisfied. As an example, suppose the following formula is used:

$$
\cos 2^{n+1} y=2\left(\cos 2^{n} y\right)^{2}-1 .
$$

If $\sin y \leq .0003162$, then $\cos y=1.0000000$ (the error being less than $\left.5 \times 10^{-8}\right)$ and $\cos 2^{n} y=1.0000000$ for all $n$; this is obviously incorrect. A better formula to use is

$$
\cos 2^{n+1} y=\left(\cos 2^{n} y\right)^{2}-\left(\sin 2^{n} y\right)^{2} .
$$

By defining the quantities

$$
F(n, y)=\cos 2^{n} y+\sin 2^{n} y
$$

and

$$
G(n, y)=\cos 2^{n} y-\sin 2^{n} y,
$$

then

$$
\cos 2^{n+1} y=F(n, y) \cdot G(n, y)
$$

and

$$
\sin 2^{n+1} y=F(n, y)^{2}-1 .
$$

Although the above formulations are clumsy, they are necessary in order that the calculation can be made on the IBM 605 calculator. The programming for an unmodified 605 is given at the end of the article. All threedigit storage units are put on an 8-6 assignment and one full sweep of program steps is impulsed. The quantities $\sin y$ and $\cos y$ are read into Factor Storage 1, 2, 3 and 4 and the answer is read out of the third through tenth positions of the counter. During computation, F1, 2 is used to store $\sin 2^{n+1} y$ and $F(n, y) ; \mathrm{F} 3,4$ is used to store $\cos 2^{n+1} y ; Z_{n}$ is stored in G1, 2 and $b_{n}$ in G3. The following modifications would save program steps but would require additional tabulator wiring. General storage 1 and 2 can be cleared by the tabulator instead of on program step 10 to set $Z_{0}=0$. The quantity $b_{0}=N$ may be read from a card or emitted from the tabulator into G3. If the tube located at panel 1-7T is removed, the absolute value of a number may be read in on one program step instead of taking three. If the final multiplication is carried out on a separate card cycle, steps 50 


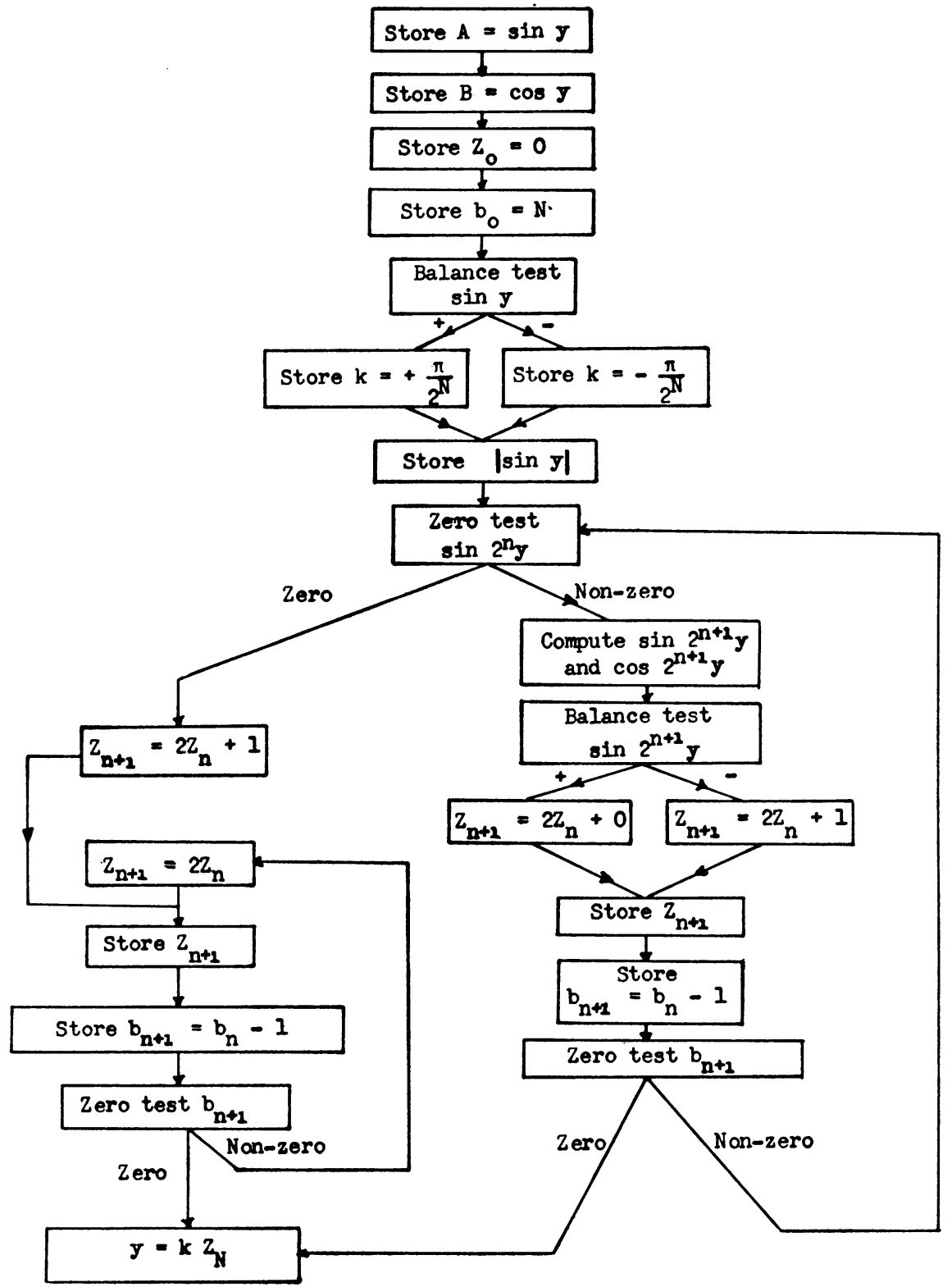

FIG. 2.

through 60 may also be eliminated. This programming allows $y$ to be any value in the region $-\pi<y<\pi$, but excludes the possibility $y=\pi$.

In the worst cases, this method requires a little over two seconds which in CPC operation is less than six card cycles. This compares favorably with the time required by other methods to attain the same accuracy, and unlike most other methods does not require extra card cycles for determining the quadrant of the answer. 
605 PROGRAMMING

\begin{tabular}{|c|c|c|c|c|c|}
\hline \multicolumn{4}{|c|}{ Step } & \multicolumn{2}{|l|}{$\begin{array}{l}\text { Suppress } \\
\text { on }\end{array}$} \\
\hline 1 & Emit 2 & MQRI & Ri 2nd & 1 & \\
\hline 2 & MQRO & Emit 6 & Prog. Exp No. 1 & 1 & Program expansion No. 1 \\
\hline $\begin{array}{l}3 \\
4\end{array}$ & $\begin{array}{l}\text { F12RO } \\
\text { P. U. Rpt. }\end{array}$ & $\begin{array}{l}\text { ECRI- } \\
\text { P.U. Rpt. }\end{array}$ & $\begin{array}{l}\text { Bal. Test } \\
\text { Reset }\end{array}$ & $11^{1}$ & is $\mathrm{G} 3 \mathrm{RI}, \mathrm{Ri}$ 6th \\
\hline & No. 1 & Del. & & & \\
\hline $\begin{array}{l}5 \\
6\end{array}$ & $\begin{array}{l}\text { F12RO } \\
\text { ECRO }\end{array}$ & $\begin{array}{l}\text { ECRI+ } \\
\text { F12RI }\end{array}$ & & $1,-$ & \\
\hline 7 & & Zero Test & Reset & & \\
\hline $\begin{array}{l}8 \\
0\end{array}$ & F12RO & ECRI+ & Bal. Test & 1 Non zero & \\
\hline 10 & & G12RI & P. U. Gp. Sup. 1 & $\begin{array}{c}1,1 \mathrm{NOI} 2 \mathrm{LiO} \\
1\end{array}$ & \\
\hline $\begin{array}{l}11 \\
12\end{array}$ & $\begin{array}{l}\text { F34RO } \\
\text { ECRO }\end{array}$ & $\begin{array}{l}\text { ECRI+ } \\
\text { MQRI }\end{array}$ & $*$ & & $\begin{array}{l}\text { * D.O. Rpt. Del. if Repeat } \\
\text { selector one is up. }\end{array}$ \\
\hline 13 & ECRO & G4RI & Ro 6th & & \\
\hline $\begin{array}{l}14 \\
15\end{array}$ & $\begin{array}{l}\text { F12RO } \\
\text { F12RO }\end{array}$ & $\begin{array}{l}\text { ECRI - } \\
\text { ECRI - }\end{array}$ & & & \\
\hline 16 & ECRR & F12RI & & & \\
\hline 17 & F12RO & Mult+ & & & \\
\hline $\begin{array}{l}18 \\
19\end{array}$ & $\begin{array}{l}\text { ECRR } \\
\text { G4RO }\end{array}$ & $\begin{array}{l}\text { F34RI } \\
\text { MORI }\end{array}$ & Ro 6th & & \\
\hline $\begin{array}{l}19 \\
20\end{array}$ & F12RO & Mult+ & & & \\
\hline 21 & F34RO & $\mathrm{ECRI}+$ & & & \\
\hline 22 & $\frac{1}{2} \operatorname{adj}_{\mathrm{CR}}$ & F34 RI & $\begin{array}{l}\mathrm{Ri} 2 \mathrm{nd} \\
\mathrm{RO} 3 \mathrm{rd}\end{array}$ & & \\
\hline 24 & Emit 1 & ECRI+ & &,- 2 & \\
\hline $\begin{array}{l}25 \\
26\end{array}$ & $\begin{array}{l}\text { G12RO } \\
\text { G12RO }\end{array}$ & $\begin{array}{l}\text { ECRI+ } \\
\text { ECRI+ }\end{array}$ & & & \\
\hline $\begin{array}{l}27 \\
28\end{array}$ & & G12RI & & Non zero & \\
\hline 29 & Sup 2 & ECRI+ & & & \\
\hline 30 & ECRO & MQRI & & & \\
\hline 31 & ECRR & G4TRI & Ro 6th & & \\
\hline $\begin{array}{l}32 \\
33\end{array}$ & $\begin{array}{l}\text { F12RO } \\
\text { ECRR }\end{array}$ & $\begin{array}{l}\text { Mult+ } \\
\text { F12RI }\end{array}$ & Ro 6th & & \\
\hline 34 & G3RO & ECRI+ & Ri 6th & & \\
\hline 35 & G4RO & G3RI & Ri 6th & & \\
\hline 37 & G3RO & $\begin{array}{l}\text { ELRI+ } \\
\text { MQRO }\end{array}$ & F12RI & & \\
\hline $\begin{array}{l}38 \\
30\end{array}$ & G4RO & MQ̆RI & & & \\
\hline 40 & $\begin{array}{l}\text { FI2RU } \\
\frac{1}{2} \text { adj. }\end{array}$ & Mult+ & Ri 2nd & & \\
\hline 41 & Emit 1 & MQRI & Ri 5th & & \\
\hline $\begin{array}{l}42 \\
43\end{array}$ & $\begin{array}{l}\text { ECRO } \\
\text { G } 3 R O\end{array}$ & $\begin{array}{l}\text { G3RI } \\
\text { ECRI- }\end{array}$ & $\begin{array}{l}\text { Ro 6th } \\
\text { Ri 6th }\end{array}$ & & \\
\hline 44 & MQRO & ECRI - & Ri 6th & & \\
\hline 45 & ECRR & F12RI & Ro 3rd & & \\
\hline 47 & G & Zero Test & Ri 6th & & \\
\hline 48 & ECRR & G3RI & & & \\
\hline $\begin{array}{l}49 \\
50\end{array}$ & $\begin{array}{l}\text { F12RO } \\
\text { Emit } 4\end{array}$ & $\begin{array}{l}\text { ECRI- } \\
\text { F34RI }\end{array}$ & $\begin{array}{l}\text { Prog. Rpt. } \\
\text { Ri 2nd }\end{array}$ & $\begin{array}{l}\text { Zero } \\
\text { Non zero }\end{array}$ & \\
\hline 51 & Emit 8 & Prog. $\operatorname{Exp} 2$ & Ri 2nd & Non zero & $\begin{array}{l}\text { Program expansion } 2 \text { is } \\
\text { ECRI }+, R i \text { th }\end{array}$ \\
\hline 52 & Emit 3 & $\mathrm{ECRI}+$ & Prog. Exp 3 & Non zero & Program expansion 3 is \\
\hline 53 & Emit 1 & ECRI+ & Ri 5th & Non zero & \\
\hline 55 & G12RO & $\widetilde{*}$ & & $\begin{array}{l}\text { Non zero } \\
\text { Non zero }\end{array}$ & ${ }^{*}$ Multiply plus if repeat \\
\hline $\begin{array}{l}56 \\
57\end{array}$ & $\begin{array}{l}\text { F34RO } \\
\text { FCRR }\end{array}$ & MQRI & Emit 6 & Non zero & selector 1 is normal, \\
\hline 58 & F34RO & $\mathrm{ECRI}+$ & & Non zero & \\
\hline 60 & $\begin{array}{l}\text { GILRU } \\
\frac{1}{2} \text { adj. }\end{array}$ & & Ri 2nd & $\begin{array}{l}\text { Non zero } \\
\text { Non zero }\end{array}$ & $\begin{array}{l}\text { Multiply plus it repeat } \\
\text { selector } 1 \text { is normal, } \\
\text { minus if transferred }\end{array}$ \\
\hline
\end{tabular}




\begin{tabular}{l}
\multicolumn{1}{c}{ Steps } \\
$8,11-13$ \\
$14-16$ \\
$17-23$ \\
$24-27$ \\
$29-45$ \\
$46-48$ \\
$50-60$
\end{tabular}

U. S. Naval Ordnance Plant Indianapolis 18 , Indiana
Description of Operation

Compute $\cos x-\sin x$ and store

Compute $\cos x+\sin x$ and store

Compute $\cos 2 x$ and store

Compute $Z_{n+1}$ and store

Compute $\sin 2 x$ and store

Modify $b_{n}$

Multiply $Z_{N}$ by $\pi / 2^{N}$

RoBERT L. LAFARA

${ }^{1} \mathrm{D}$. TEICHROEw, "Use of continued fractions in high speed computing," $M T A C$, v. 6,

${ }^{2}$ C. HAstings, Approximations in Numerical Analysis. (Form 15's). Rand Corporation, Santa Monica. Sheets 8-12, 35-37, 39.

${ }^{3}$ W. P. Heising, "An eight-digit general-purpose control panel," IBM Tech. News Letter No. 3, 1951.

${ }^{4}$ D. W. SweEnEy, "A Model II four address floating-decimal coding system," IBM Tech. News Letter No. 5, 1953.

\section{A Partitioning Method of Inverting Symmetric Definite Matrices on a Card-Programmed Calculator}

Although certain standard lines of approach seem to exist in all methods of inverting matrices, the problem of inversion has not yet been solved with finality, and modifications on "well-worn" solutions often prove to be of value. This paper discusses a partitioning method for inverting matrices which is particularly suited to the International Business Machines' Card Programmed Calculator, (C.P.C.), and subsidiary equipment.

The general problem is to invert an $n^{\text {th }}$ order matrix

$$
M=\left(\begin{array}{ll}
a & b \\
c & d
\end{array}\right)
$$

where $a$ and $d$ are each square submatrices of order $n_{1}$ and $n_{2}\left[n_{1}+n_{2}=n\right]$. Let the inverse of $M$ be

$$
M^{-1}=\left(\begin{array}{cc}
A & C \\
B & D
\end{array}\right)
$$

partitioned in the same manner as the original matrix. Post-multiplying $M$ by its inverse, we have

$$
\left(\begin{array}{ll}
a A+b B & a C+b D \\
c A+d B & c C+d D
\end{array}\right)=\left(\begin{array}{ll}
1 & 0 \\
0 & 1
\end{array}\right)
$$

or in equation form

$$
\begin{array}{ll}
a A+b B=1 & \text { (identity matrix of order } \left.n_{1}\right) \\
c A+d B=0 & \left(\text { zero matrix } n_{2} \times n_{1}\right) \\
a C+b D=0 & \left(\text { zero matrix } n_{1} \times n_{2}\right) \\
c C+d D=1 & \text { (identity matrix of order } \left.n_{2}\right)
\end{array}
$$

